\title{
Console for the Rapid and Precise Comparison of Volt Boxes
}

\author{
Patrick H. Lowrie, Jr.* \\ Institute for Basic Standards, National Bureau of Standards, Boulder, Colo.
}

(March 24, 1966)

\begin{abstract}
The comparison of volt boxes within uncertainties of \pm 0.01 percent or less has, in the past, been a long and difficult task at the NBS Boulder Laboratories. A console for this purpose recently put in use has reduced the time and labor to a great extent and, at the same time, has reduced the uncertainties associated with the measurement circuitry to less than 0.001 percent, thus allowing realizable calibration uncertainties of less than 0.005 percent. The console was designed as part of a system in which the operator was considered to be the decision-making link in the measurement chain. The circuitry, through the use of a contained computer and associated circuits, provides the operator with the data he requires in the form he chooses. The operator is thus freed from the need to consider minutiae, and thereby is able to concentrate on those factors requiring judgment.
\end{abstract}

Key Words: Calibration, console, electrical, measurement, ratio, standards, voltage, volt box.

\section{Introduction}

A volt box is a carefully constructed voltage divider having a number of fixed ratios between input and output terminals. These ratios are commonly in multiples of the integers 1 through 5 . A volt box is normally used to extend the effective range of a potentiometer so that voltages up to 750 or $1500 \mathrm{~V}$ may be measured.

As a volt box is a ratio device, the absolute resistance of its elements is not of great importance and under ordinary circumstances need be known only in a nominal sense (i.e., $750 \Omega / \mathrm{V}$, etc.). However, before a volt box may be used for accurate measurements, the actual ratio must be determined. There are two characteristics inherent in volt boxes that manifest themselves in a dependence of the ratio upon the applied voltage. One is the change in the resistance of the elements with heating. In the large "master" volt boxes fabricated in accordance with NBS RP1419 $[1],{ }^{1}$ the changes in ratio resulting from self-heating are usually quite small, being in the order of 5 to 20 ppm (parts per million) at rated voltage. In the less complex, general purpose boxes, however, the difference between the ratio at reduced voltage (under $30 \%$ of rated) and that at rated voltage may be as great as $200 \mathrm{ppm}$. The other characteristic is the variation in the resistance of the insulating parts of the box. Even when using the new, high-resistance insulating materials, small currents across the surface of the insulator can result from surface contamination. When these currents are in parallel with resistance paths, the ratio is disturbed. These currents are not, in general, a linear function of applied voltage, and

*Radio Standards Laboratory, National Bureau of Standards, Boulder, Colo.

${ }^{1}$ Figures in brackets indicate the literature references on page 180. the current paths cannot be well-defined. To reduce the effects of these currents, the more complex, master boxes have guarded circuits wherein the voltage across the leakage path is reduced considerably with a corresponding reduction in leakage currents.

When these characteristics are considered, it becomes apparent that the ratio should be measured at the voltage for which the box will be used. This may be done most easily by comparing the box with another volt box having known characteristics. Two methods of comparison are described in RP1419. (The second method is discussed in appendix I of that paper.) The first method is the one used at NBS.

The recently completed console used for this measurement at the NBS Boulder Laboratories incorporates many new features that contribute to the accuracy and efficiency of the measurement. With this console, uncertainties associated with the measurement circuitry are less than 0.001 percent, which allows realizable calibration uncertainties of less than 0.005 percent. This paper describes the console and includes, as appendices, an evaluation of the uncertainties that may be expected in its use.

\section{Comparison Measurement}

As a comprehensive description of this measurement may be found in the literature, [2] only a brief treatment will be undertaken here. The circuit is shown in figure 1. In the course of the measurement, the values of $v_{3}, v_{2}, v_{1}$, and $v_{4}$ are measured in microvolts. $\quad\left(v_{3}\right.$ is normally measured first because it is the voltage most sensitive to changes in ratio caused by heating and to wrong connections in the measuring circuit.) When these values and the supply voltage, $V$, are known, it can be shown [3] that the corrected ratio of the box 


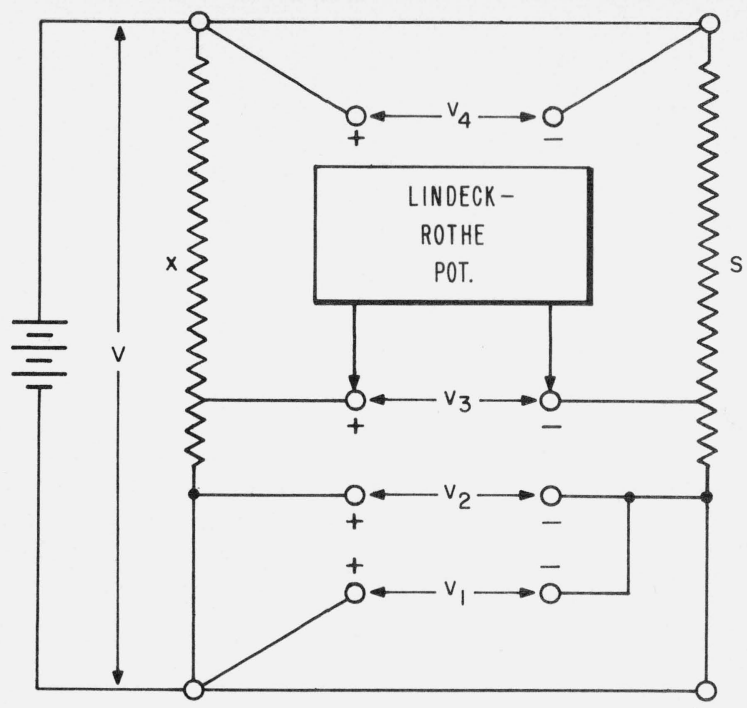

FIGURE 1. Basic volt box measurement circuit.

undergoing comparison (within the limits of uncertainty in the measurement) is

$$
X=X_{n}\left[1+\left(\mu_{s}+\frac{v_{4}-v_{1}}{V}+\frac{v_{2}-v_{3}}{V / S_{n}}\right) 10^{-6}\right]
$$

where $X_{n}$ is the nominal ratio of the unknown,

$\mu_{s}$ is the correction to the ratio of the standard volt box in ppm,

$S_{n}$ is the nominal ratio of the standard volt box, and is equal to $X_{n}$,

$V$ is the supply voltage, in volts, and

$v_{1}, v_{2}, v_{3}$, and $v_{4}$ are the measured voltages, in microvolts.

$\left(v_{1}, v_{2}\right.$, and $v_{3}$ are considered to be positive when of the same polarity as $v_{4}$.)

\section{Measuring System Requirements}

In order to achieve a high degree of accuracy, the measuring system must, of course, be fabricated of stable, high-quality components and must be guarded. However, the use of good quality equipment alone is not a guarantee of highly accurate measurements. The success of the measurement is dependent to a very large extent upon the operator. Human operational factors contributing to degradation of accuracy are primarily fatigue, both muscular and nervous, and inability to copy figures indefinitely without error. In the design of the console, these factors were carefully considered, and as a result the following requirements were formulated: (1) The operator should be able to change ratios quickly and easily. This will not only reduce irritation but also will minimize the time during which no power is applied and thus reduce uncontrolled cooling of the volt boxes. It is important to minimize this cooling because volt boxes are calibrated under temperature equilibrium conditions. (2) Controls should be centralized as much as possible. (3) The digital form of indication is to be preferred over the analog form. (4) All readings and settings should be capable of being checked. (5) As much of the computing as possible should be done automatically. (In the calibration of a volt box eq (1) may have to be calculated as many as 32 times.) (6) Recopying of data should be reduced to a minimum.

In addition to the requirements listed above, the fact that voltages as high as $1500 \mathrm{~V}$ dc may be present during calibration dictates the use of adequate safety measures.

\section{Features}

The console was designed from the human engineering viewpoint to as great an extent as was technically and economically practical. The operator was treated as a decision maker, and the console was designed to automatically handle those functions not requiring decision and to supply the operator with the appropriate data in a clear and concise form (fig. 2). As a result of this viewpoint, and of the requirements listed above, the following features were included. (1) A plug-panel was designed to allow the operator to quickly change ratios on the standard volt box. (2) All controls are grouped in front of the operator. During the measurement of a given ratio, the operator does not need to leave his seat, and no control is more than 18 in. away. However, the operator does need

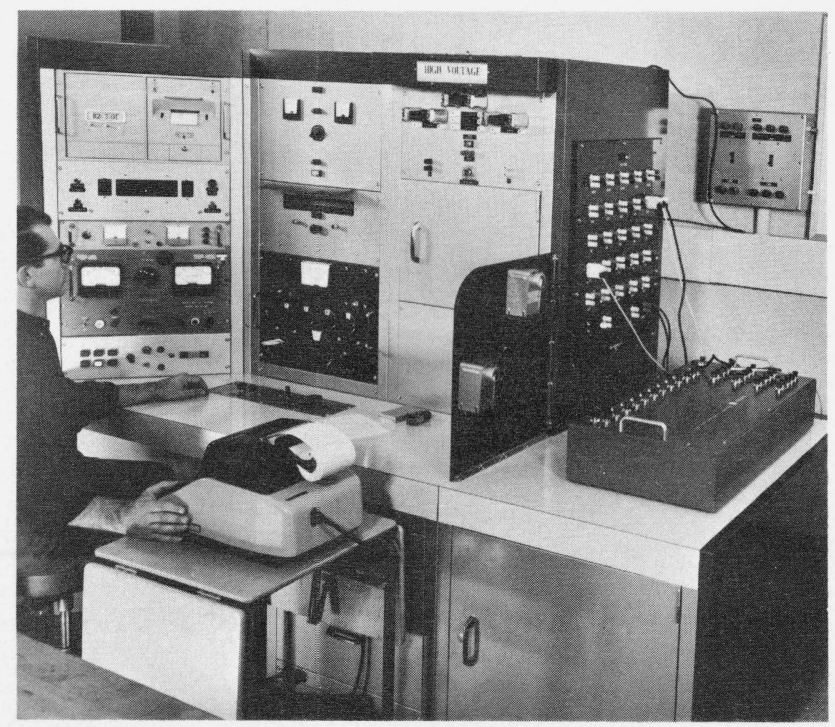

Figure 2. The volt box calibration console at the NBS Boulder Laboratories, now at NBS laboratories, Gaithersburg, Md. 


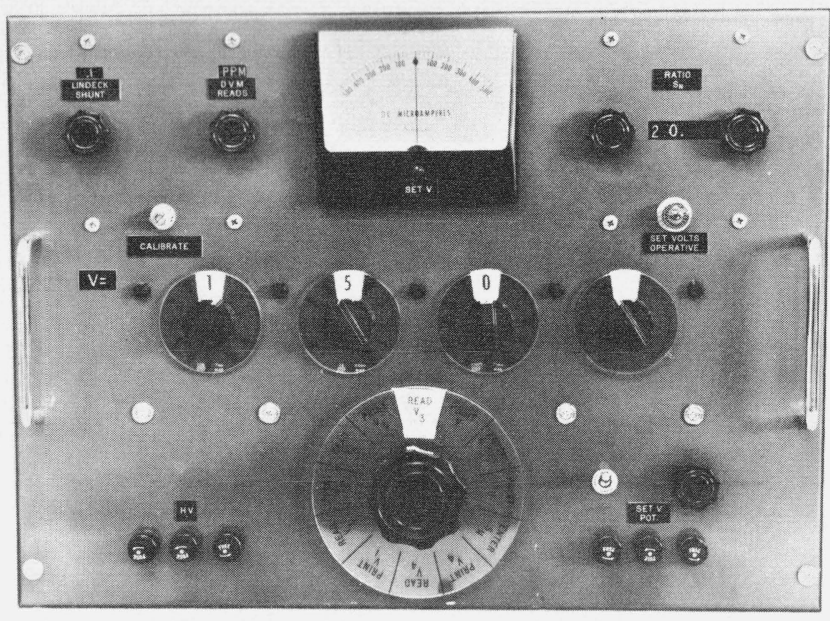

A

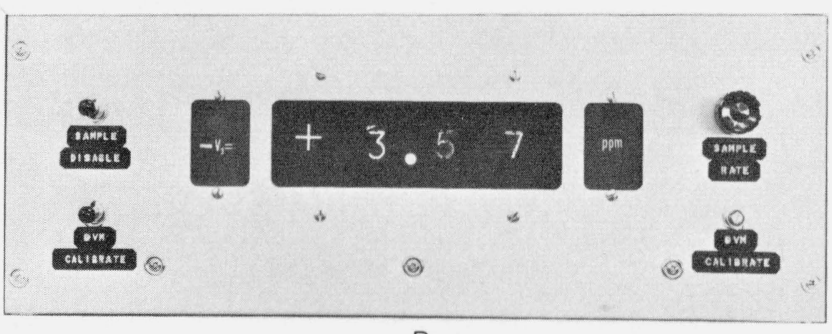

B

FigURE 3. A. Main control panel; B. Output display panel.

to get up and move around the end of the console to change ratios. (3) All important control settings and displays are in digital form (fig. 3). (4) The basic measuring circuit includes provision for reducing the measured voltage to parts per million continuously and automatically. The value, in ppm, is recorded by the operator on an adding machine. (5) The tape from the adding machine, when complete, is cut into appropriate lengths and photographically reproduced on $8 \frac{1}{2} \times 11$ inch sheets which become the permanent records. Thus the data need be recopied by hand only once, onto the summary sheet from which the Report of Calibration is typed. To comply with the safety requirements, the area in which the high voltage is exposed is protected by a two-stage photoelectric fence. If the illumination to either of two photocells is interrupted, the high voltage is disconnected. Also, a prominently mounted, high-voltage sign is illuminated whenever the high voltage circuit is energized. To provide an indication of high voltage even if all other safety circuits were to fail, two neon bulbs are connected, in series with suitable dropping resistors, across the high voltage leads. One of these is mounted on the front panel, and the other is mounted at the top of the plug-panel in the high-voltage area.

Initially a digital printer was connected to print the position of every control on the main control panel and the output from the measuring circuit. The tape from the printer was to be used to check the validity of the recorded data. However, the action of the printer adversely affected the measuring circuit, and it proved to be impractical to reduce this circuit interaction to a tolerable level. The use of the printer was therefore discontinued. The checking of recorded data is accomplished by the making of multiple measurements.

\section{Measurements Procedure}

In the measurement of a given range of a volt box, the operator first connects the box as shown in figure 2 . The main function switch (the large, rotary switch in the lower center of the main control panel, fig. 3A) is rotated to the "Enter $S_{n}$ " position. The operator enters the nominal value of the ratio into the console circuitry by adjusting the switches in the upper right of the main control panel. He then rotates the main function switch to the "Enter and Set $V$ " position. (In so doing he moves the switch through the "Print $S_{n}$ " position which originally caused the printer to record the values of the $S_{n}$ dials. This position no longer has any meaning.) After having turned the four " $V=$ " dials to the desired value of applied voltage (the fourth dial controls the position of the decimal point), he adjusts the high-voltage supply until a null is obtained on the Set- $V$ meter. By rotating the main function switch to the "Read $v_{3}$ " position, he makes those circuit connections necessary for the measurement of the difference voltage shown in figure 1 . He records the values of $V$ and $S_{n}$ on the calculator, and then proceeds to measure $v_{3}$, then $v_{2}, v_{1}$, and $v_{4}$. As the measurement of each difference voltage is completed, he enters the value displayed on the output display panel (fig. 3B) into the calculator. After having completed the four measurements, he enters the correction to the standard into the calculator. By depressing the totaling key, he completes the calculations.

\section{Computer Principle}

If eq (1) is rewritten as shown in eq (2), the correction to the ratio of the box undergoing calibration is seen to be the sum of five terms, each of which is in parts per million, provided the conditions listed under eq (1) are met.

$$
X=X_{n}\left[1+\left(\mu_{s}+\frac{v_{4}}{V}-\frac{v_{1}}{V}+\frac{S_{n} v_{2}}{V}-\frac{S_{n} v_{3}}{V}\right) 10^{-6}\right] .
$$

As only multiplication and division are needed to convert the measured voltage to parts per million, an electrical analog is easily constructed. Considering the $v_{3}$ term of eq (2),

$$
V_{3(\mathrm{ppm})}=v_{3(\mu V)} \frac{S_{n}}{V}
$$


Correspondingly, in a voltage divider circuit,

$$
E_{0}=E \frac{R_{2}}{R_{1}}
$$

where $R_{2}$ is the portion of $R_{1}$ across which $E_{0}$ appears. It remains only to determine the circuit that allows the relationship

$$
\frac{E_{0}}{E}=\frac{R_{2}}{R_{1}}=\frac{S_{n}}{V}
$$

to hold, considering that $V$ and $S_{n}$ are independent parameters.

If $R_{2}$ is allowed to vary in such a way as not to change the value of $R_{1}$, the two values will be independent and eq (5) may be realized in fact. This is done as is explained in section 7.3. The result is a simple, completely passive computer, the accuracy of which is limited only by the accuracy of the contained resistors.

In the actual design, no attempt was made to retain the decimal point in the voltage divider circuit, as that would require excessive voltages in the computer. Instead, a separate switching circuit is used to place the decimal point.

\section{Circuit}

\subsection{Block Diagram}

Figure 4 shows a block diagram of the circuit. The output from the $0-1500 \mathrm{~V}$ supply is monitored by the set-volts potentiometer. This potentiometer is also used to enter the significant figures of the value of $V$ (eq (2)) into the computing potentiometer such that $R_{1}$ of eq (5) is proportional to $V$. The significant figure of $S_{n}$ is similarly entered by adjusting the value of $R_{2}$ in eq (5) appropriately. Decimal information is supplied to the decimal point computer as shown. (The value of $V$ may be anywhere between 0 and $1500 \mathrm{~V}$, to three significant figures. That of $S_{n}$ may lie anywhere between $1 . \times 10^{\circ}$ and $9 . \times 10^{4}$, but in practice it does not exceed $1 \times 10^{4}$.) The four difference voltages $\left(v_{1}, v_{2}, v_{3}\right.$, and $\left.v_{4}\right)$ to be measured are connected to the computing potentiometer by means of a relay switching circuit. The output from the computing potentiometer is connected to a digital volt-meter which has been modified to read only significant figures. The decimal information proceeds directly to the digital display from the decimal point computer. The printer monitored both the computing potentiometer (which included, for the purpose of printing, the settings on the set-volts and $S_{n}$ dials) and the digital display. The printer output included codes indicating the nature of the display (ppm, microvolts, etc.) as well as the value and sign.

\subsection{Set-Volts Potentiometer}

\section{a. Significant Figures Section}

The set-volts potentiometer circuit is shown in figure 5 . The significant figures section comprises a one-volt zener source supplying a Kelvin-Varley voltage divider. Each decade of the divider is $1000 \Omega$ per step with $2500 \Omega$ resistors used for compensation. This type of design allows all decades to be of a high (and easily procured) resistance which reduces the

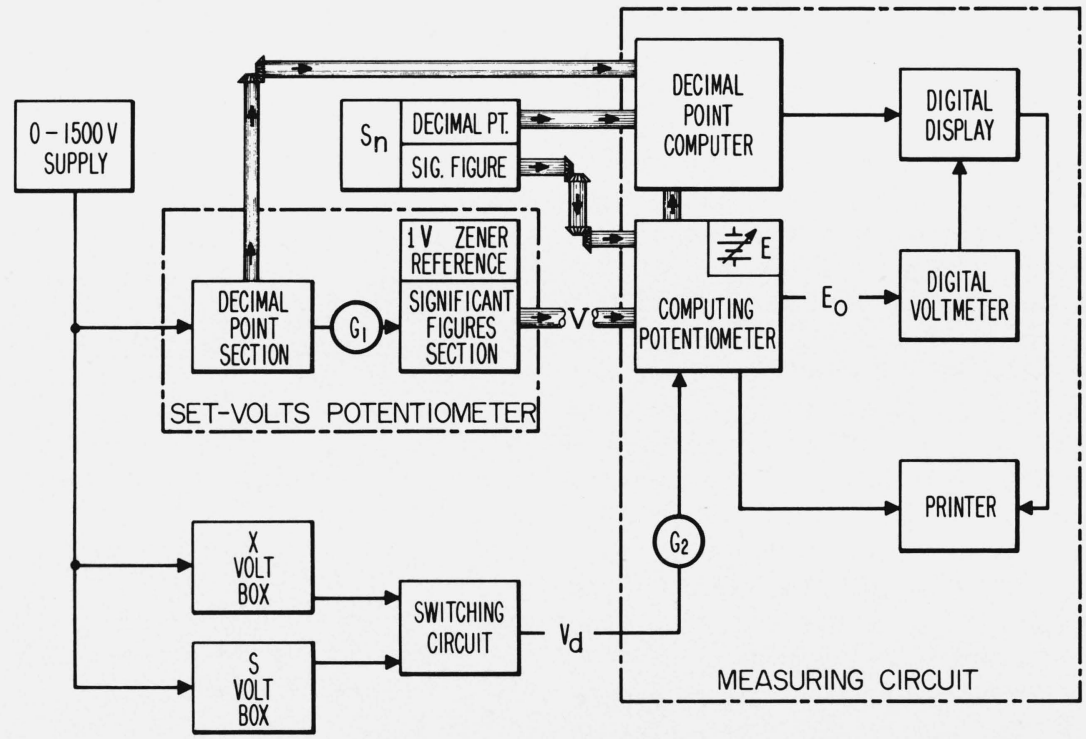

FigURE 4. Block diagram of console circuit. 


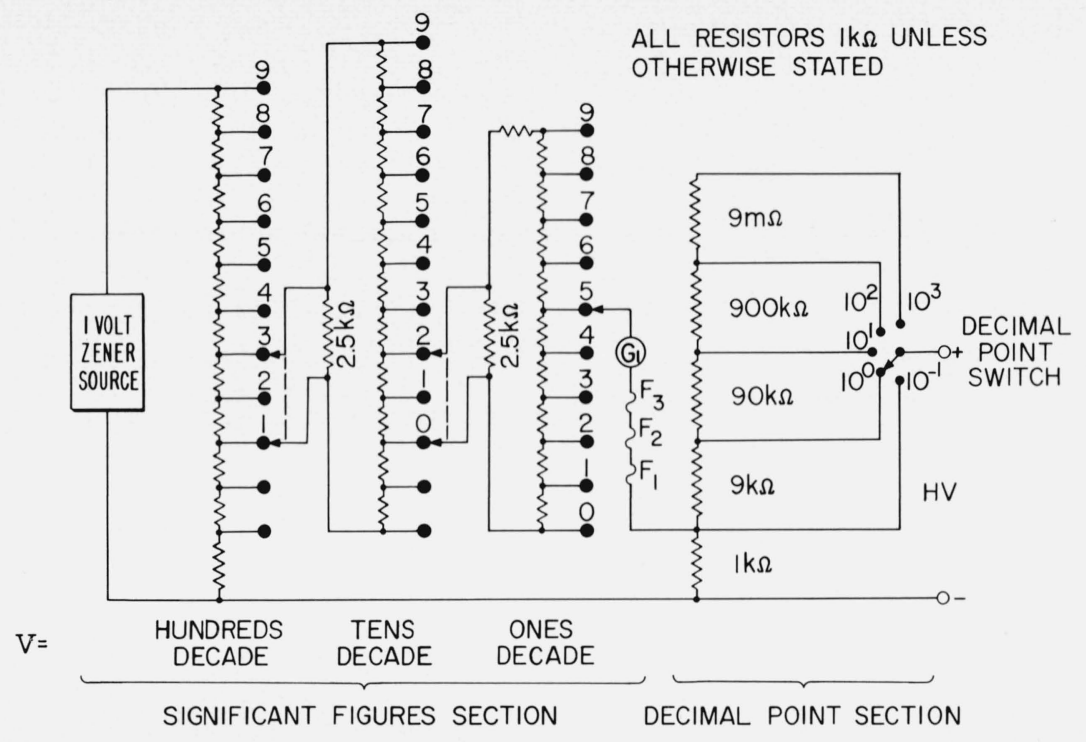

FigurE 5. The set-volts potentiometer.

effect of contact resistance. Each decade is physically ganged to, but guarded and shielded from, a corresponding decade in the computing potentiometer. Thus, as the value of $V$ is set on the three decades in the set-volts potentiometer, the value of $R_{1}$ in eq (5) is simultaneously adjusted to be proportional to $V$. It should be noted that the significant figures of the lowest value that can be set on the set-volts potentiometer dials are 100 . This is necessitated by the need, in the computer, for $R_{1(\min )}=10 \mathrm{k} \Omega>R_{2(\max )}$ $=9 \mathrm{k} \Omega$. A more detailed explanation is given in section 7.3.

It will be noted that the galvanometer, $G_{1}$ (which is of the chopper-amplifier type), is protected by three fuses $\left(f_{1}, f_{2}\right.$, and $\left.f_{3}\right)$ in series. The possibility, however remote, of the operator inadvertently applying $1500 \mathrm{~V}$ directly across the galvanometer circuit necessitated this, and the fusing system has been found to be entirely adequate.

\section{b. Decimal Point Section}

The decade voltage divider connected across the high-voltage terminals divides that voltage by integral powers of ten. The switch used for this purpose is the fourth switch to the right in the " $V=$ " section on the main control panel (fig. 3). This switch also enters the appropriate power of ten in the Decimal-Point computer and lights the appropriate decimal point light on the main control panel (fig. 3). The relationship between the decimal point section of figure 5 and the " $V=$ " dials of figure 3 is as follows. The $10^{-1}$ position causes the decimal point light preceding the first significant figure to light; hence the reading of the $V=$ section, as shown in the figure, would be .150. The $10^{\circ}$ position causes the light between the first and second significant figures to light; hence the reading would be 1.50 , etc. When the decimal point switch is rotated to the $10^{3}$ position, a zero appears in the fourth window of the $V=$ section and the decimal point light to the right of that window will light. With the switch in this position the reading would be 1500 . If a potential of $1500 \mathrm{~V}$ was now impressed across the decimal-point-section divider, the voltage to the right of $G_{1}$ would be

$$
\frac{1500 .\left(10^{3}\right)}{10^{7}}=0.150 \text { volt }
$$

which is equal to the voltage to the left, and the galvanometer would be at null.

The possible errors introduced by the circuits in the set-volts potentiometer are discussed in appendix A.

\subsection{Computing Potentiometer}

The computing potentiometer (fig. 6) is basically a modified Lindeck-Rothe circuit connected to a voltage divider. The ammeter normally used as an indicator has been replaced by a $10 \mathrm{k} \Omega$ resistor. Hence the output from the potentiometer is a voltage rather than a meter deflection. As is explained below, this voltage is operated on by the voltage divider comprising $R_{1}$ and $R_{2}$ such that

$$
E_{0}=\frac{R_{2}}{R_{1}} M \cdot v_{d},
$$




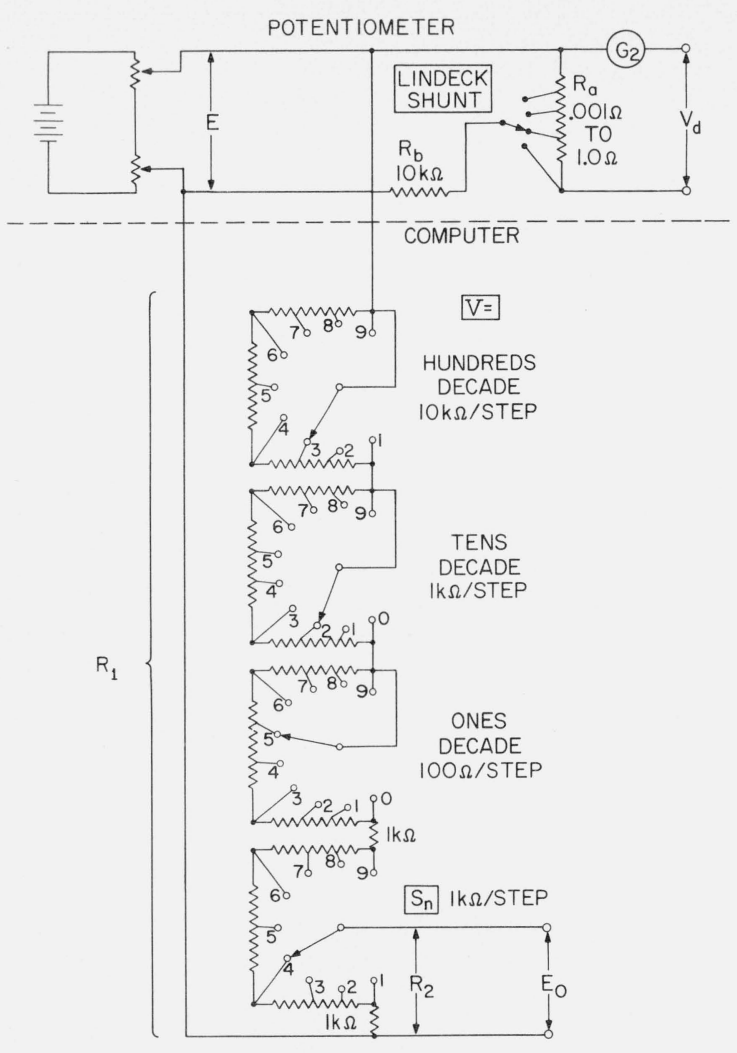

Figure 6. Computing potentiometer. the " $V=$ " dials are adjusted to 325 , for example, the resistance of $R_{1}$ becomes $32.5 \mathrm{k} \Omega$ and the result is shown in figure $7 \mathrm{~b}$. The value of $E_{0}$ in figure $7 \mathrm{~b}$ is equal to that of figure 7 a divided by 325 (ignoring the decimal point). Thus the " $V=$ " value is entered as a divisor. If the $S_{n}$ decade is adjusted to 4 , the circuit is as shown in figure $7 \mathrm{c}$, and $E_{0}$ is four times that of figure $7 \mathrm{~b}$. Thus the value of $S_{n}$ is entered as a multiplier, and the circuit of figure 6 meets the requirement of eq (5).

The errors associated with the computing potentiometer are discussed in appendix B.

\subsection{Decimal Point Computer}

The decimal point is placed by means of the circuit shown in figure 8 . Placement is determined by three switches, as shown. It will be noted that as the value of the Lindeck shunt selected is increased, the decimal point moves to the right. This is true also when the $S_{n}$ decimal point switch is changed to select a larger value. If the decimal point switch on the set-volts potentiometer is adjusted to a larger value, however, the decimal point on the digital display will move to the left. Thus the Lindeck shunt and $S_{n}$ decimal point switches serve to multiply by powers of 10 , and the set-volts decimal point switch divides by powers of 10. In the actual circuit, provision is included for bypassing the $S_{n}$ switch when $v_{1}$ and $v_{4}$ are being measured.

\subsection{Switching Circuit}

where $v_{d}$ is the voltage to be measured by the potentiometer, and $M$ is a multiplier determined by the values of $R_{a}$ and $R_{b}$.

The three decades shown under " $V=$ " in figure 6 are ganged to the corresponding decades in the setvolts potentiometer. It will be noted that when these decades are adjusted to the minimum value $(V=100)$ there is still $10 \mathrm{k} \Omega$ of resistance in the circuit. It is this feature that allows $R_{2}$ of eq (5) to vary independently of $R_{1}$.

When both the " $V=$ " and " $S_{n}$ " decades are adjusted to minimum, the circuit is as shown in figure $7 \mathrm{a}$. If
The measuring circuit is connected to the voltages $v_{1}, v_{2}, v_{3}$, and $v_{4}$ by means of double-pole, single-throw relays, as shown in figure 9 . It will be noted that the movable and stationary contacts of any relay are separately guarded. This is necessary to minimize the effects of leakage currents flowing between the contacts when the relay is deenergized. With the circuit as shown, any leakage currents of this type flow directly from the high-voltage supply and hence have no effect on the ratio. To implement the guarding shown in figure 9, it was necessary to modify commercially available relays to the form shown in figure 10 .

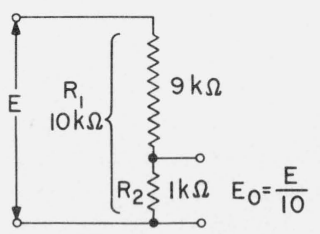

A

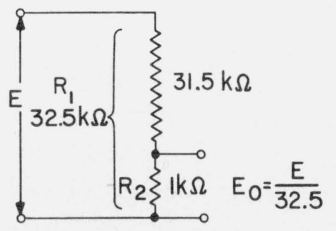

B

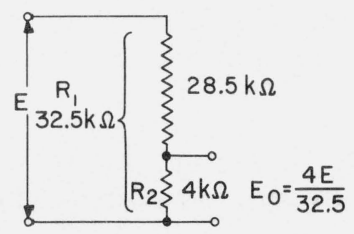

C

FigURE 7. Computer circuit showing "V=" and " $\mathrm{S}_{\mathrm{n}}$ " adjustments.

$$
\begin{aligned}
& \text { A: } V=100, S_{n}=1 \\
& \text { B: } V=325, S_{n}=1
\end{aligned}
$$

C: $V=325, S_{n}=4$. 
GANGED TO

LINDECK SHUNT SWITCH

$\mathrm{S}_{2}$

GANGED TO

DECIMAL POIN

SWITCH ON

SET VOLTS

POTENTIOMETER

$\mathrm{S}_{3}$

GANGED TO

$S$ DECIMAL

POINT SWITCH

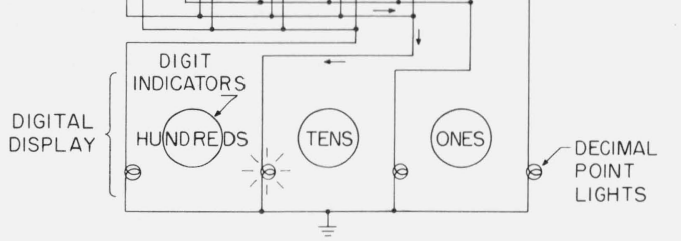

Figure 8. Decimal point computer (simplified).

\subsection{Guard Circuit}

In keeping with personnel safety requirements, the cabinets of the console are connected to a low-impedance ground. Grounding the cabinets also provides the measuring circuit with shielding. The measuring circuit guard is grounded also as shown in figure 9. The power supplies are isolated; one side of the high-voltage line is grounded (see fig. 9). When $v_{1}, v_{2}$, or $v_{3}$ is being measured, the side connected to $v_{1}$ is grounded. When $v_{4}$ is measured, the side connected to $v_{4}$ is grounded.

\section{Conclusion}

The incorporation of this console into the measurement facility at the Boulder Laboratories has resulted in a significant reduction in the limits of uncertainty associated with the calibration of volt boxes. The results of measurements made on a box of the type described in RP14.19 are well within the predicted worst-case uncertaintly of $\pm 8 \mathrm{ppm}$ (see appendix C).

TABLE 1. Comparison of measurements made by the self-check method with those made on the console

\begin{tabular}{|c|c|c|c|c|c|}
\hline \multirow{3}{*}{$\begin{array}{c}A \\
\text { Range }\end{array}$} & \multicolumn{4}{|c|}{ Corrections in ppm } & \multirow{3}{*}{$\begin{array}{c}\text { Comparison } \\
\text { in ppm } \\
\\
\stackrel{F}{E-B}\end{array}$} \\
\hline & \multirow{2}{*}{$\begin{array}{c}B \\
\text { Self check }\end{array}$} & \multicolumn{2}{|c|}{ Console measurements } & \multirow{2}{*}{ Ave of $C \& D$} & \\
\hline & & $C$ & $D$ & & \\
\hline $3 / 1.5$ & -2 & +5 & +1 & +3 & +5 \\
\hline $4.5 / 1.5$ & -4 & 0 & +1 & 0 & +4 \\
\hline $6 / 1.5$ & -6 & 0 & 0 & 0 & +6 \\
\hline $7.5 / 1.5$ & -7 & -4 & -1 & -2 & +5 \\
\hline $15 / 1.5$ & -9 & -8 & -7 & -8 & +1 \\
\hline $30 / 1.5$ & -9 & -9 & -10 & -10 & -1 \\
\hline $45 / 1.5$ & -7 & -6 & -6 & -6 & +1 \\
\hline $60 / 1.5$ & -7 & -5 & -6 & -6 & +1 \\
\hline $75 / 1.5$ & -7 & -4 & -6 & -5 & +2 \\
\hline $150 / 1.5$ & -3 & -3 & -2 & -2 & +1 \\
\hline $300 / 1.5$ & -1 & 0 & 0 & 0 & +1 \\
\hline $450 / 1.5$ & +2 & +1 & 0 & 0 & -2 \\
\hline $600 / 1.5$ & +1 & +1 & +1 & +1 & 0 \\
\hline $750 \quad / 1.5$ & +1 & +3 & +3 & +3 & +2 \\
\hline
\end{tabular}

These results are shown in table 1. Column B shows the results of measurements made by the "self checking" technique described in appendix II of RP1419. Columns C and D are the results of two independent measurements made by comparing the unknown (previously self-checked) volt box with the standard by means of the console and, in fact, reflect not only the console uncertainties, but the uncertainties in the correction to the standard ratio and any uncertainties introduced by the operator also. The comparisons were made at 20 percent of rated voltage on each range to approximate the conditions under which the self check was made. Column $\mathrm{E}$ is the average of columns C and D. These conclusions are corroborated by the results of a second experiment that involved measurements of three master volt boxes in the following manner.

Figure 10. Switching circuit relay. 
First V.B. 1 was measured against V.B. 2, then V.B. 3 was measured against V.B. 1, and finally V.B. 3 was measured against V.B. 2.

In the first and third measurements, V.B. 2 was considered the absolutely perfect standard. In the second measurement, V.B. I was considered the absolutely perfect standard.

If the corrections, in ppm, to $\mathrm{V}$. B. 1 resulting from the first measurement are called $A$; those to V. B. 3 resulting from the second measurement are called $B$; and those to V. B. 3 resulting from the third measurement are called $C$, the following relationship should exist [5].

$$
A+B-C=0
$$

provided the corrections to the three volt boxes did not change between measurements, and provided no errors are introduced by the measuring system -in this case, the console. To insure unchanging corrections in the volt boxes, measurements were made at reduced voltage. When the data from the measurements were applied to eq (8), the results were as shown in figure 14.

The author is indebted to Joseph J. Barth, who deciphered the many schematics and wiring diagrams and reduced them to operating hardware.

\section{References}

[1] Silsbee, F. B., and Gross, F. J., Testing and performance of volt boxes, J. Res. NBS 27, 269-287 (Sept. 1941) RP1419.

[2] Dunfee, B. L., Method for calibrating a standard volt box, J. Res. NBS 67C (Engr. and Instr.) No. 1, 1-3 (Jan.-Mar. 1963).

[3] Op. Cit., p. 2.

[4] Dunn, A. F., Calibration of a Kelvin-Varley Voltage Divider, 1964 IEEE International Convention Record, Part 8, Sessions on Instrumentation, pp. 213-224.

[5] Youden, W. J., Uncertainties in calibration, IRE Trans. Instr. Vol. I-II, Nos. 3 and 4, December 1962, pp. 134-135.

\section{Appendix A. Evaluation of the Set-Volts Potentiometer}

\subsection{Kelvin-Varley Circuit (See Fig. 5)}

The rigorous mathematical analysis of a KelvinVarley divider is quite complex, and has appeared in the literature.[4]

As the divider in the Set-Volts Potentiometer is to be used without applying corrections, the maximum effective uncertainty that will be introduced by the divider is the only thing that must be determined. Individual errors in the several sections are important only in their contribution to the overall error. As a recently calibrated digital voltmeter having combined uncertainties within 0.01 percent was available for the purpose, it was simpler and easier to measure this

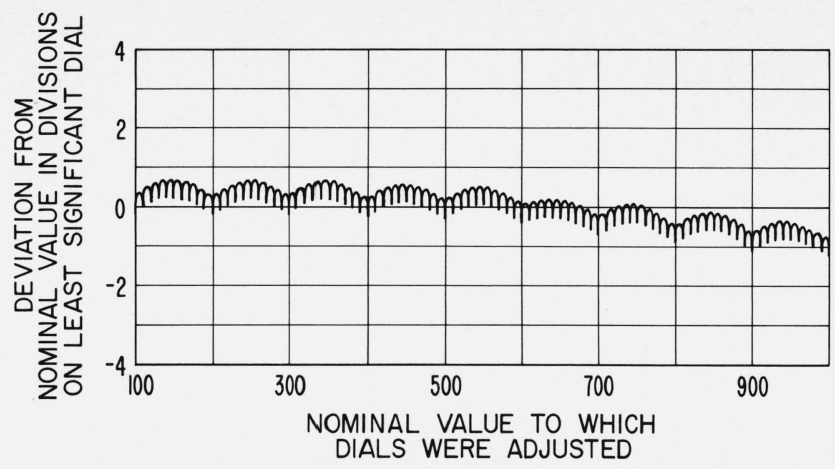

Figure 11. Measured deviations of $K-V$ divider ratio from dial settings.

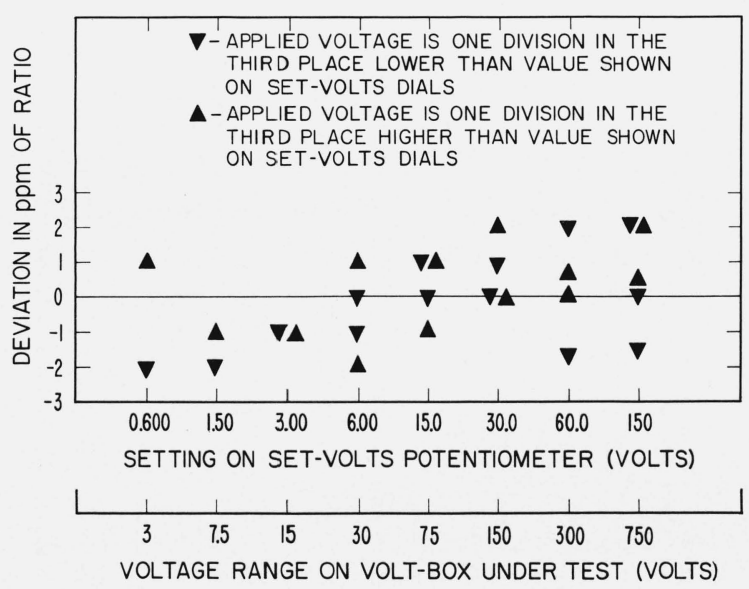

Figure 12. Deviations in measured value of ratio caused by disagreement between value of applied voltage and value to which set- $V$ dials are set.

overall error than to calculate it. Accordingly, the 900 settings on the set-volts divider were measured against the digital voltmeter, and the data were checked at 19 points with a commercial potentiometer (in which the combined uncertainties were within $0.01 \%$ ). The results of these measurements are shown in figure 11. As can be seen, the largest error introduced by the nonlinearity of the divider is roughly equal to one digit on the third (least significant) dial. When the applied voltage is adjusted to a value equal to one division in the third place higher or lower than the value to which the Set-Volt Potentiometer dials are set, the resulting difference in measured ratio, from that determined when the applied voltage and dial setting are equal, is smaller than the random errors encountered in the measurement. The results of such an experiment are shown in figure 12. 


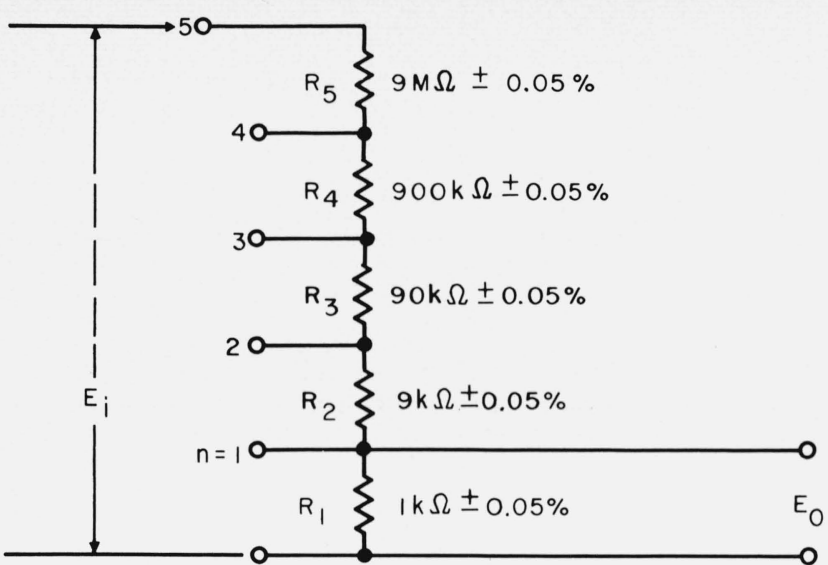

FIGURE 13. Decimal divider in set-volts potentiometer.

\subsection{Decimal Divider}

The decimal divider is shown in figure 13, which corresponds to the circuit of the Decimal Point Section in figure 5. In figure 13, the relationship of $E_{0}$ to $E_{i}$ for any given switch setting, $n$, is

$$
E_{0}=\left(\frac{R_{1}}{R_{1}+R_{2}+\ldots+R_{n}}\right) E_{i}
$$

where $E_{0}$ and $R_{1}, R_{2}, \ldots R_{n}$ are the nominal values. The true value, $E_{0}^{\prime}$, however, is

$$
E_{0}^{\prime}=E_{0}\left(1+e_{a}\right)=\frac{R_{1}\left(1+r_{1}\right) E_{i}}{\sum_{1}^{n} R_{k}\left(1+r_{k}\right)} .
$$

Dividing eq (A2) by eq (A1) gives

$$
1+e_{a}=\frac{\left(1+r_{1}\right) \sum_{1}^{n} R_{k}}{\sum_{1}^{n} R_{k}\left(1+r_{k}\right)},
$$

or

$$
e_{a}=\frac{\left(1+r_{1}\right) \sum_{2}^{n} R_{k}-\sum_{2}^{n} R_{k}\left(1+r_{k}\right)}{\sum_{1}^{n} R_{k} \cdot\left(1+r_{k}\right)},
$$

which reduces to

$$
e_{a}=\frac{\sum_{2}^{n} R_{k}\left(r_{1}-r_{k}\right)}{\sum_{1}^{n} R_{k}\left(1+r_{k}\right)} .
$$

Equation (A5) will be maximum when $n=5$ and $r_{2}, r_{3}$, $r_{4}$, and $r_{5}$ are opposite in sign to $r_{1}$. In this worst case, assuming the corrections to all the resistors are maximum, i.e., $0.0005,{ }^{2} e_{a}$ is found to be

$$
e_{a} \doteq 0.001 \text {. }
$$

\section{Appendix B. Evaluation of Errors Asso- ciated With the Computing Potentiometer}

As explained in the text, the milliameter of the Lindeck-Rothe potentiometer has been replaced by a resistor $\left(R_{b}\right)$ of $10,000 \Omega$ nominal value (see fig. 6). The voltage across $R_{b}$ will (nominally) be $10^{4}, 10^{5}$, $10^{6}$, or $10^{7}$ times $v_{d}$ depending on which value of $R_{a}$ is switched into the circuit. If this value, $V_{R_{b}}$, is applied across the divider circuit, $R_{1}$, the nominal value of voltage, $E_{0}$, applied to the digital voltmeter would be found by the relation:

$$
E_{0}=\frac{R_{2}}{R_{1}} \frac{R_{b}}{R_{a}} v_{d}
$$

${ }^{2}$ The resistors used were individually measured and found to have corrections well within the limits of \pm 0.0005

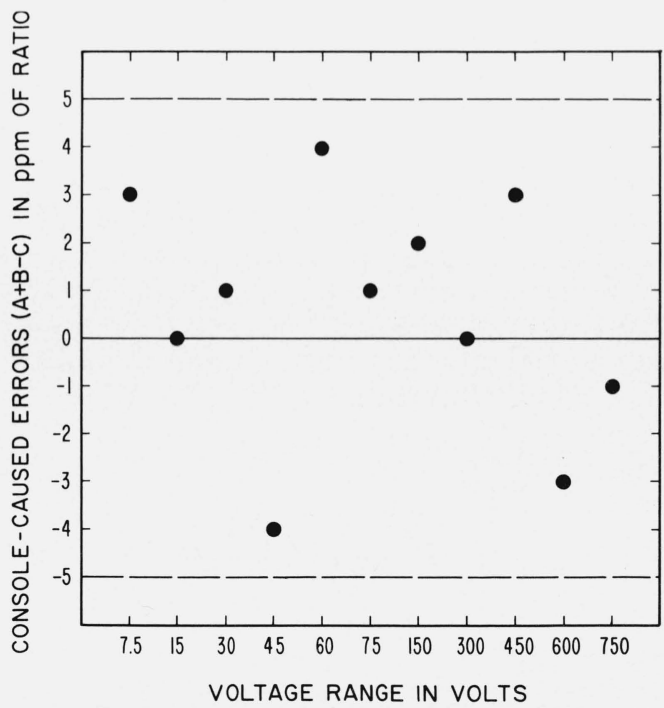

FIGURE 14. Console-caused errors as determined by intercompari son of three master volt boxes. 
where $v_{d}$ is any one of the four difference voltages $\left(v_{1}, v_{2}, v_{3}\right.$, or $\left.v_{4}\right), R_{1}$ and $R_{2}$ are the displayed (i.e., nominal) values proportional to $V$ and $S_{n}$ respectively, see eq (1), $R_{b}$ is nominally $10,000 \Omega$, and $R_{a}$ is nominally either $1,0.1,0.01$, or $0.001 \Omega$ as determined by the position of the Lindeck Shunt switch. How ever, it is not practicable to measure $V_{R_{b}}$ without changing its effective value (because of the loading effect of the measuring system) and hence disturbing the ratio of multiplication, $R_{b} / R_{a}$. Therefore, to prevent this disturbance, the voltage is measured across $R_{b}+R_{a}$. With a circuit of this configuration, the voltage applied across $R_{1}$ is not $V_{R_{b}}$, but $V_{R_{b}}+V_{R_{a}}$, and the actual value, $E_{0}^{\prime}$, is related to $v_{d}$ by the equation

$$
E_{0}^{\prime}=\frac{R_{2}^{\prime}}{R_{1}^{\prime}} \frac{R_{b}^{\prime}+R_{a}^{\prime}}{R_{a}^{\prime}} v_{d}
$$

In eq (B2) the primes indicate actual (as opposed to nominal) values.

As providing automatic correction for the effect of $R_{a}$ in the numerator of eq (B2) would have appreciably increased the cost of the console, it was decided to treat the circuit as though the voltage applied across $R_{1}$ is $V_{R_{b}}$ even though in fact this is not the case. This will, of course, introduce an error in addition to that caused by the errors in $R_{1}, R_{2}, R_{a}$, and $R_{b}$. It is the purpose of this appendix to determine the relative error $\left(e_{0}\right)$ in $E_{0}$, for any given $v_{d}$, resulting both from the errors in the component values and from the presumption that eq (Bl) is the circuit equation.

If the circuit of figure 6 is examined rigorously it will be found that the actual value of each of the components differs, although slightly, from the nominal value. If we consider each actual value (designated by a prime) to be comprised of the nominal value and the small difference between nominal and actual (i.e., $R_{a}^{\prime}=R_{a}\left(1+r_{a}\right)$ ), the circuit equation for determining the actual output voltage for a given $v_{d}$ is

$$
E_{0}^{\prime}=E_{0}\left(1+e_{0}\right)=\frac{R_{2}\left(1+r_{2}\right)}{R_{1}\left(1+r_{1}\right)}\left[\frac{R_{b}\left(1+r_{b}\right)+R_{a}\left(1+r_{a}\right)}{R_{a}\left(1+r_{a}\right)}\right] v_{d}
$$

where $e_{0}, r_{1}, r_{2}, r_{a}$, and $r_{b}$ are the relative differences between the actual values, $E_{0}^{\prime}, R_{1}^{\prime}, R_{2}^{\prime}, R_{a}^{\prime}$, and $R_{b}^{\prime}$ and the nominal values $E_{0}, R_{1}, R_{2}, R_{a}$, and $R_{b}$, respectively.

Equation (B3) may be more readily analyzed if it is considered to be of the form

$$
E_{0}\left(1+e_{0}\right)=T_{1}\left(1+t_{1}\right) T_{2}\left(1+t_{2}\right) v_{d} .
$$

If, in eq (B4), the following relations hold,

$$
\begin{gathered}
\mathrm{T}_{1}\left(1+t_{1}\right)=\frac{R_{2}\left(1+r_{2}\right)}{R_{1}\left(1+r_{1}\right)}, \\
T_{2}\left(1+t_{2}\right)=\frac{R_{b}\left(1+r_{b}\right)+R_{a}\left(1+r_{a}\right)}{R_{a}\left(1+r_{a}\right)},
\end{gathered}
$$

and

$$
T_{1}=\frac{R_{2}}{R_{1}}
$$

$$
\mathrm{T}_{2}=\frac{R_{b}}{R_{a}}
$$

then eq (B4) may be rewritten as

$$
E_{0}\left(1+e_{0}\right)=\frac{R_{2}}{R_{1}} \frac{R_{b}}{R_{a}}\left(1+t_{1}\right)\left(1+t_{2}\right) v_{d} .
$$

From a comparison of eq (B9) and eq (B1) it is immediately apparent that

$$
1+e_{0}=\left(1+t_{1}\right)\left(1+t_{2}\right) .
$$

It is now possible to examine each of the component errors, $t_{1}$ and $t_{2}$, individually; this possibility greatly facilitates the analysis.

Let us first determine the relative error, $t_{2}$. If we divide eq (B6) by eq (B8), the result is

$$
1+t_{2}=\frac{R_{a}}{R_{b}}+\frac{1+r_{b}}{1+r_{a}}
$$

If $\left|r_{a}\right|$ and $\left|r_{b}\right|$ are small (i.e., $\left.<0.001\right)$, eq (B11) reduces approximately ${ }^{3}$ (i.e., to first degree terms) to

$$
t_{2} \doteq \frac{R_{a}}{R_{b}}+r_{b}-r_{a}
$$

In the circuit, the resistors $R_{a}$ and $R_{b}$ have been chosen such that $\left|r_{a}\right|<0.0004$ and $\left|r_{b}\right|<0.0001$. From figure 6 it will be seen that

$$
\frac{R_{a}}{R_{b}} \leqslant 0.0001
$$

Thus $t_{2}$ will be within the limits

$$
\left|t_{2}\right| \leqslant\left(\frac{R_{a}}{R_{b}}\right)_{\max }+\left|r_{a}\right|+\left|r_{b}\right|=0.0006 .
$$

The evaluation of $t_{1}$ may be handled in a similar manner. The division of eq (B5) by eq (B7) gives

$$
1+t_{1}=\frac{1+r_{2}}{1+r_{1}}
$$

\footnotetext{
${ }^{3}$ If $\left|r_{a}\right|$ and $\left|r_{b}\right|$ are each less than 0.001 , the difference between the right-hand side of eq (B12) and the true value,

$$
t_{2}=\frac{R_{a}}{R_{b}}+\frac{1+r_{b}}{1+r_{a}}-1
$$

$$
\left|\frac{r_{a}\left(r_{b}-r_{a}\right)}{1+r_{a}}\right|<2 \times 10^{-6}
$$

and hence is so small as to not significantly affect the value of $t_{2}$.
}

is 
If $\left|r_{1}\right|$ and $\left|r_{2}\right|<0.001$, eq (B15) becomes

$$
t_{1} \doteq r_{2}-r_{1} \text {. }
$$

From figure $6, R_{1}$ is seen to comprise many resistors in series. These resistors and switches have been chosen so that the actual value of the resistance added by any decade, on any position, does not differ from the nominal value by more than \pm 0.03 percent. Also the two $1 \mathrm{k} \Omega$ resistors have been similarly chosen. Hence the circuit of $R_{1}$ may be realistically represented by eq (B17). (As $R_{1}$ is the total resistance of the network, it is represented by $R_{t}$ in the following analysis.)

$$
R_{t}\left(1+r_{t}\right)=\sum_{k=1}^{n} R_{k}\left(1+r_{k}\right),
$$

where $R_{1}, R_{2}, \ldots, R_{n}$ are the resistors in the $S_{n}$ switch, the $1 \mathrm{k} \Omega$ resistors connected to either end of that switch, and all other resistors that have been switched into the circuit by the settings of the three " $V=$ " dials. Equation (B17) may be rewritten in the form

$$
R_{t}\left(1+r_{t}\right)=\sum_{1}^{n} R_{k}+\sum_{1}^{n} R_{k} r_{k}
$$

Since

$$
R_{t}=\sum_{1}^{n} R_{k}
$$

eq (B18) may be divided by $R_{t}$ to give the equation

$$
r_{t}=\frac{1}{R_{t}} \sum_{1}^{n} R_{k} r_{k}=\left(\sum_{1}^{n} R_{k} r_{k}\right) /\left(\sum_{1}^{n} R_{k}\right)
$$

That is, $r_{t}$ is dependent upon the sum of the differences, in ohms, between the actual and nominal values of the resistors $R_{k}$ in ratio with the sum of the nominal values. If, as previously stated, all values of $\left|r_{k}\right|$ are within some limit, $r$, then the value, $r$, as substituted for $\left|r_{k}\right|$ may be factored out of eq (B20) to yield the bounds to $r_{t}$; i.e.,

$$
\left|r_{t}\right| \leqslant r \frac{\sum_{1}^{n} R_{k}}{\sum_{1}^{n} R_{k}}=r .
$$

Therefore, the bounds to $r_{t}$ (i.e., to the relative difference between the actual and nominal values of $R_{1}$ in fig. 6) are 0.0003 . Similar reasoning ${ }^{4}$ can be used to determine the bounds to the relative error of $R_{2}$ (fig. 6) with the result:

$$
\left|r_{2}\right| \leqslant 0.0003 \text {. }
$$

Hence, the bounds to $t_{1}$ are found, using eq (B16), to be

$$
\left|t_{1}\right| \leqslant 0.0006 \text {. }
$$

However, the validity of this value is questionable since the interdependence of the errors of $R_{1}$ and $R_{2}$ was not considered in the analysis. A more rigorous analysis results when the resistance network $R_{1}$ is considered to comprise the independent resistances $R_{3}$ and $R_{2}$ such that

$$
R_{3}+R_{2}=R_{1} .
$$

If this is done, eq (B5) becomes

$$
T_{1}\left(1+t_{1}\right)=\frac{R_{2}\left(1+r_{2}\right)}{R_{3}\left(1+r_{3}\right)+R_{2}\left(1+r_{2}\right)} .
$$

The determination of the error introduced by $R_{3}$ and $R_{2}$ may be found by operating on the relation

$$
\frac{R_{2}}{R_{3}+R_{2}}\left(1+e_{r}\right)=\frac{R_{2}\left(1+r_{2}\right)}{R_{3}\left(1+r_{3}\right)+R_{2}\left(1+r_{2}\right)} .
$$

Dividing eq (B26) by $R_{2} /\left(R_{3}+R_{2}\right)$ gives

$$
1+e_{r}=\frac{\left(R_{3}+R_{2}\right)\left(1+r_{2}\right)}{R_{3}\left(1+r_{3}\right)+R_{2}\left(1+r_{2}\right)} .
$$

If $\left|r_{3}\right|$ and $\left|r_{2}\right|<0.001$ (this has already been demonstrated as the reasoning used in the determination of $r_{t}$ applies with equal validity to $r_{3}$ ), eq (B27) may be approximated by (the reasoning of footnote 4 applies)

$$
1+e_{r} \doteq \frac{R_{3}+R_{2}}{R_{2}+R_{3}\left(1+r_{3}-r_{2}\right)}
$$

being more than adequately accurate for the purpose intended. Subtracting 1 from both sides allows eq (B28) to be reduced to the more usable form,

$$
e_{r}=\frac{R_{3}\left(r_{2}-r_{3}\right)}{R_{2}+R_{3}\left(1+r_{3}-r_{2}\right)} .
$$

From eq (B29), $\left|e_{r}\right|$ approaches the value

$$
\frac{r_{2}-r_{3}}{1+r_{3}-r_{2}}
$$

${ }^{4} \mathrm{As} R_{2}$ is in parallel with the input impedance of the digital voltmeter, the actual re sistance may differ from nominal by as much as 0.09 percent unless compensating techniques are used. These techniques, in the form of trimmer resistors, were used on $R_{2}$. Hence the resistances shown are the effective values including the input impedance of the Hence the resistances shown are the effective values including
digital voltmeter, adjusted to within \pm 0.03 percent $(300 \mathrm{ppm})$. 
as a maximum limit as the ratio $R_{3} / R_{2}$ increases without bound. Using bounds of \pm 0.0003 for $r_{3}$ and $r_{2}$

$$
\left|e_{r}\right| \leqslant \frac{0.0003+0.0003}{1-.0003-.0003} \text {. }
$$

Hence

$$
\left|e_{r}\right| \leqslant 0.0006 \text {. }
$$

A comparison of statement (B32) with statement (B23) shows that (B23) is indeed valid.

Referring again to eq (B4), the value, $e_{0}$, is quite approximately determined by the relation

$$
e_{0} \doteq t_{1}+t_{2}
$$

provided $\left|t_{1}\right|$ and $\left|t_{2}\right|$ are $<0.001$. That these values are less than 0.001 has been demonstrated. Therefore $e_{0}$ may be determined from eq (B33) (using the limits of $t_{1}$ and $t_{2}$ listed in statements (B14) and (B23)) to be

$$
\left|e_{0}\right| \leqslant|0.0006|+|0.0006|=0.0012 \text {. }
$$

This figure, found by direct addition, is certainly the pessimistic limit in the worst case (i.e., where $R_{1} / R_{2} \rightarrow \infty$ ). A more realistic appraisal might be to combine the different independent errors by the square root of the sum of the squares, as they are as likely to compensate for one another as to add. If this is done using eq (B12) and (B16), then with high probability

$$
\left|e_{0}\right| \leqslant \sqrt{r_{1}^{2}+r_{2}^{2}+\left(R_{a} / R_{b}\right)^{2}+r_{b}^{2}+r_{a}^{2}}
$$

the result is

$$
\left|e_{0}\right| \leqslant 0.0006 \text {. }
$$

The actual limits of $e_{0}$ will lie somewhere between these two extremes.

Hence the premise of eq (B1) is found to be uncertain by about \pm 0.1 percent of $E_{0}$.

Total Measurement Circuit Uncertainty. An additional uncertainty of \pm 0.2 percent $\left(e_{m}\right)$ of the measured voltage is introduced by the digital voltmeter. Also the digital display has an inherent uncertainty of \pm one digit in the last place. Because of the way in which the display is used, this \pm one digit causes an absolute uncertainty $\left(e_{d}\right)$ of $\pm 1 \mathrm{ppm}$ of the ratio (of the unknown volt box) in the worst case for each displayed value.

\section{Appendix C. Evaluation of the Measure- ment Uncertainty of the Volt Box Console}

This evaluation does not include the effect of the uncertainties associated with the corrections to the Standard Volt Box.
The equation of correction for the unknown volt box as used by the operator of the console is

$$
X_{n}\left[1+(x) 10^{-6}\right]=X_{n}\left[1+\left(\mu_{s}+V_{1}+V_{2}+V_{3}+V_{4}\right) 10^{-6}\right],
$$

where $X_{n}$ is the nominal ratio of the unknown, $x$ is the correction to the unknown in ppm, $V_{1}, V_{2}, V_{3}$, and $V_{4}$ are the computed results in ppm, and $\mu_{s}$ is the correction to the standard volt box in $\mathrm{ppm}$.

The error in $V_{1}, V_{2}, V_{3}$, or $V_{4}$ is closely approximated by the relation:

$$
e \approx e_{v}-e_{V}
$$

where $e_{v}$ is the error resulting from the computer and $e_{V}$ is the error caused by the set-volts potentiometer. The difference in errors shown in eq (C2) is the reason that the zener supply for the set-volts potentiometer need only be calibrated against the digital voltmeter. If the digital voltmeter is in error (absolutely) by some value, the set-volts potentiometer will be in error by the same value, and the two errors will subtract to zero.

The uncertainties in $e$ resulting from the dividers in the set-volts potentiometer, the resistors in the computing circuits, etc., must be considered as the signs of these uncertainties are not known.

$e_{v}$ and $e_{V}$ are the actual values of the errors and, of course, are not known. It is possible to determine the approximate bounds within which these values must fall.

The error, $e_{v}$, is comprised of the contributing errors $e_{0}$ (the error in the voltage measured by the digital voltmeter), $e_{m}$ (the error introduced by the digital voltmeter circuits), and $e_{d}$ (the uncertainty in the last digit of the display) (see appendix B). These components have been determined to have the limits

$\left|e_{0}\right| \leqslant 0.0012$ of voltage measured by the digital voltmeter

$\left|e_{m}\right| \leqslant 0.0002$ of voltage measured by the digital voltmeter

$e_{d}= \pm 1 \mathrm{ppm}$ of measured ratio.

The values of the limits of $\boldsymbol{e}_{0}$ and $\boldsymbol{e}_{m}$ are functions of the voltage that is measured by the digital voltmeter and can be combined. Since $e_{0}$ and $e_{m}$ are independent errors, their uncertainties can be combined by the relation:

$$
\left|e_{v}^{\prime}\right| \leqslant \sqrt{e_{0}^{2}+e_{m}^{2}}
$$

to give the highly probable limits to this portion of $e_{v}$. Thus

$$
\begin{gathered}
\left|e_{v}^{\prime}\right| \leqslant \sqrt{(0.0012)^{2}+(0.0002)^{2}} \\
\left|e_{v}^{\prime}\right| \leqslant 0.0012
\end{gathered}
$$


However, there is a possibility that the component errors could, under some circumstances, be of such sign that they would add, giving a value approximating the simple sum of the limits of uncertainty. Under these conditions

$$
\left|e_{v}^{\prime \prime}\right| \leqslant 0.0014
$$

The value of $e_{d}$ must be treated separately.

The error $e_{V}$, is also comprised of component errors. These are $e_{a}$, the error resulting from the divider of figure 13, (see appendix A) and the errors introduced by the Kelvin-Varley divider.

The value of $e_{a}$ has been determined to be within the limits \pm 0.0001 of the applied voltage, $V$. The errors resulting from the Kelvin-Varley divider are somewhat obscure, but conservatively may be assumed to lie within the limits $\pm 2 \mathrm{ppm}$ of the measured ratio.

The accuracies of the values of $V_{1}, V_{2}, V_{3}$, and $V_{4}$ are directly dependent upon the accuracy with which the applied voltage can be measured (see eqs (2) and (3) in the text). However, the error in the measurement of the applied voltage will be the same for each value, and, though the sign is not known, it will be the same also. Hence the uncertainty of the measured ratio will very probably lie within the limits

$|u| \leqslant\left[\left(V_{1} e_{v}^{\prime \prime}+V_{2} e_{v}^{\prime \prime}+V_{3} e_{v}^{\prime \prime}+V_{4} e_{v}^{\prime \prime}\right)\right.$

$$
\left.+\left(V_{1}+V_{2}+V_{3}+V_{4}\right) e_{a}+4 e_{d}+e_{k v}\right]
$$

where $e_{k v}$ is the value of the limits of the error introduced by the Kelvin-Varley divider in ppm of ratio. When the limits of the different component uncertainties are substituted in eq (C7), the limits of $u$ are found to be approximately

$$
|u| \leqslant 8 \text { ppm. }
$$

(Paper 70C3-229) 